\title{
\begin{tabular}{|c|lc|}
\hline IV Simpósio Paranaense de Modelagem, & Artigo: & 02 \\
$\begin{array}{c}\text { Simulaçãoe Controle de Processos } \\
\text { ISSN : 1984-7521 }\end{array}$ & Páginas: & $\mathbf{0 9}$ - $\mathbf{1 6}$ \\
\cline { 2 - 2 } & & Pán
\end{tabular}
}

\section{Modelagem e controle de conversores CC-CC via estratégias de controle clássicas}

\author{
Isaías V. de Bessa, Renan Landau P. de Medeiros e Florindo A. de C. Ayres Junior \\ Universidade Federal do Amazonas, Manaus-AM, Brasil
}

\begin{abstract}
The use of renewable energy has enabled new ways of generating electricity by means of photovoltaic energy, wind energy, among others. In order to improve the voltage regulation, $D C$ DC converters are used. Modeling Buck converters and linearization around an operating point ensures proper operation of the drive as a voltage regulator or a power regulator. To guarantee the operation of these converters as regulators, controllers are designed to ensure that the permanent and transient regime specifications for the converter operating at the operating point.In this work the controller designed is the PID structure being designed by two classical methodologies: Pole Placement and Root Locus. Once the controllers were designed, the performance of the controller acting with the system outside the operating point was verified.
\end{abstract}

Keywords: DC-DC Converters, Buck, regulator, Pole Placement, Root Locus.

\section{Introdução}

É comum realizar a integração de sistemas de geração de energia renovável a um dispositivo armazenador de energia, como baterias, e juntamente realizar a regulação de tensão deste sistema por meio de conversores CC-CC [1-3]. A interligação de vários sistemas de geração CC é denominado de microrrede, esta pode operar de modo isolado ou conectado à rede de distribuição [2]. Dessa forma, o responsável pela alimentação do sistema é chamado de Feeder. Uma carga conectada ao Feeder é responsável por um consumo de potência constante, esta por sua vez é chamada de Carga de Potência Constante (CPL, Constant Power Load).

A conexão do $C P L$ ao Feeder ocasiona oscilações que podem levar a instabilidade do barramento $\mathrm{CC}$, constituído na conexão do sistema. Dessa forma a utilização de controladores para a mitigação desse efeito resulta em um aumento de desempenho e estabilidade da microrrede.[4,5]

Em [6] é mostrado o comportamento do $C P L$ em uma rede de distribuição $\mathrm{CC}$, neste estudo é utilizado um atenuardor ativo na rede para a redução das oscilações. Em [7] é investigado o controle do Feeder por meio de uma metodologia de controle moderno, utilizando Linear-Quadratic Regulator. Este trabalho, estabelece uma comparação entre metodologias clássicas de controle para a mitigação dos efeitos oscilatórios da conexão entre o Feeder e o CPL.

O sistema proposto nesse trabalho, é composto por dois conversores Buck conectados em cascata. O primeiro conversor irá funcionar como o Feeder fornecendo tensão constante para o segundo conversor, de modo que o segundo conversor opere como $C P L$. Considerando que os dois conversores operam em frequências distintas, vale ressaltar que o principal objetivo é verificar as oscilações no Feeder, decorrentes da mudança de referência de potência do $C P L$, possibilitando avaliar a estabilidade do sistema com a necessidade do uso de controle para melhorar o desempenho do sistema.

O restante do trabalho está organizado como segue: Na Seção 2, é apresentada a modelagem do conversor Buck. Na Seção 3, é apresentada a metodologia para o desenvolvimento da microrrede investigada, bem como é apresentada as metodologias utilizadas para o projeto dos controladores e a descrição dos testes desenvolvidos. Na 
Seção 4 é apresentado os resultados deste estudo, avaliando e discutindo os resultados para as metodologias de controle utilizadas no sistema de microrrede investigado, por fim, na Seção 5 é aprentado as principais conclusões deste estudo.

\section{Modelagem do conversor Buck}

O conversor Buck mostrado na Figura 1a é responsável por fornecer um nível de tensão de saída, $V_{o}$, menor que o nível de tensão de entrada, $V_{i}$, através de um Duty Cycle, $D$, do conversor. Dessa forma, a análise do circuito conversor se faz em dois momentos: No intervalo $T_{o n}$, mostrado na Figura $1 \mathrm{~b}$, em que a chave estática encontra-se fechada, e no no outro intervalo $T_{\text {off }}$ em que a chave estática encontra-se aberta, como mostrado na Figura 1c.

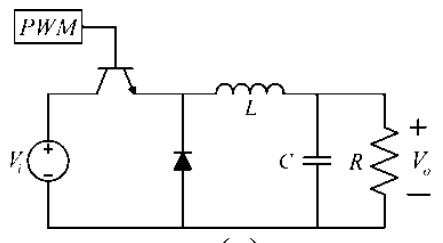

(a)

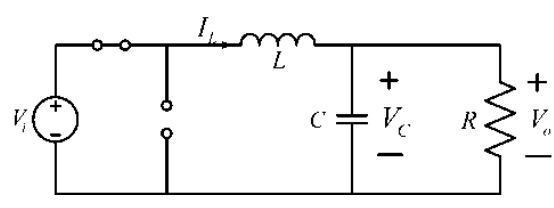

(b)

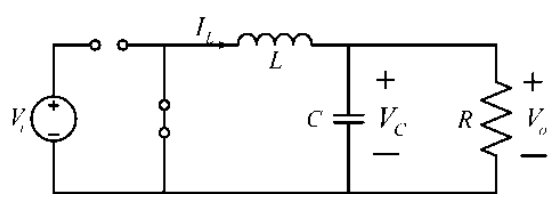

(c)

Figura 1: (a) Conversor Buck (b) para o intervalo $T_{o n}$ e (c) para o intervalo $T_{\text {off }}$

Aplicando as Lei de Kirchhoff da Tensão (LKT) e as Lei de Kirchhoff da Corrente (LKC) no circuito mostrado na Figura 1b, obtém-se as relações das Eq.(1) e Eq.(2).

$$
\begin{aligned}
& \dot{I}_{L}=-\frac{1}{L} V_{C}+\frac{1}{L} V_{i}, \\
& \dot{V}_{C}=\frac{1}{C} I_{L}-\frac{1}{R C} V_{C}
\end{aligned}
$$

Aplicando LKT e LKC no circuito mostrado na Figura 1c obtém-se as relações das Eq.(3) e Eq.(4).

$$
\begin{gathered}
\dot{I}_{L}=-\frac{1}{L} V_{C}, \\
\dot{V}_{C}=\frac{1}{C} I_{L}-\frac{1}{R C} V_{C}
\end{gathered}
$$

Utilizando o modelo de espaço de estado médio, pode-se escrever o sistema dinâmico mostrado na Eq.(5), para mais detalhes sobre espaço de estados médio consultar [8].

$$
\left[\begin{array}{c}
\dot{I}_{L} \\
\dot{V}_{C}
\end{array}\right]=\left[\begin{array}{cc}
0 & -\frac{1}{L} \\
\frac{1}{C} & -\frac{1}{R C}
\end{array}\right]\left[\begin{array}{c}
I_{L} \\
V_{C}
\end{array}\right]+\left[\begin{array}{c}
D \\
L \\
0
\end{array}\right]\left[V_{i}\right]
$$

A Eq. (5) apresenta o modelo dinâmico de um conversor Buck para um Duty Cycle invariante. Considerando que o Duty Cycle é composto por um ponto de operação fixo, $D_{o}$, e por uma parte variante no tempo $\Delta D$, pode-se considerar a relação mostrada na Eq.(6).

$$
D(t)=D_{o}+\Delta D
$$

Dessa forma o sistema descrito na Eq.(5), é considerado não-linear. Para o circuito Buck da Figura 1a operando com o Duty Cycle, $D_{o}$, no ponto de operação, irá fazer o capacitor carregar com uma tensão, $V_{C o}$, e o indutor com uma corrente, $I_{L o}$. Dessa forma, linearizando o sistema em torno do ponto $X=\left(D_{o}, I_{L o}, V_{C o}\right)$, de acordo com [8- 
9], modela-se o sistema dado na Eq.(5) para uma variação de corrente, $\Delta I_{L}$, e uma variação de tensão, $\Delta V_{C}$, deste modo pode-se reescrever o sistema dinâmico conforme apresenta a Eq.(7).

$$
\left[\begin{array}{c}
\Delta \dot{I}_{L} \\
\Delta \dot{V}_{C}
\end{array}\right]=\left[\begin{array}{cc}
0 & -\frac{1}{L} \\
\frac{1}{C} & -\frac{1}{R C}
\end{array}\right]\left[\begin{array}{c}
\Delta I_{L} \\
\Delta V_{C}
\end{array}\right]+\left[\begin{array}{c}
\frac{V_{i}}{L} \\
0
\end{array}\right][\Delta \mathrm{D}]
$$

De posse do modelo matemático do conversor Buck, torna-se possível investigar estratégias de controle que permita regular a tensão e/ou potência na saída do conversor. A regulação de tensão na saída do conversor, constitui a construção de um conversor Feeder. Pelo circuito da Figura la a saída do sistema pode ser definida conforme a Eq.(8).

$$
V_{o}=V_{C}
$$

Vale ressaltar, que o sistema foi linearizado em torno do ponto de operação $X$, e a saída do sistema é apresentado conforme apresenta a relação.

$$
\left[\Delta V_{o}\right]=\left[\begin{array}{ll}
0 & 1
\end{array}\right]\left[\begin{array}{c}
\Delta I_{L} \\
\Delta V_{C}
\end{array}\right]
$$

O sistema Feeder funcionando em seu ponto de operação, deve por sua vez alimentar uma carga, representada neste estudo pelo $C P L$. A regulação de potência na saída do conversor, constitui a construção de um $C P L$, além de considerar que a frequência de operação deste conversor é maior que a do conversor Feeder. Pelo circuito da Figura 1a a saída do sistema em potência pode ser definida pela Eq.(10).

$$
P_{o}=\frac{V_{C}^{2}}{R}
$$

Como o sistema foi linearizado em torno de $X$, pode-se escrever a saída do sistema, conforme apresentado na Eq.(11).

$$
\left[\Delta P_{o}\right]=\left[\begin{array}{ll}
0 & \frac{2 V_{C o}}{R}
\end{array}\right]\left[\begin{array}{l}
\Delta I_{L} \\
\Delta V_{C}
\end{array}\right]
$$

\section{$3 \quad$ Metodologia}

O procedimento de operação do sistema de microrrede desenvolvido, bem como os principais pontos da operação e desenvolvimento do sistema implementado está apresentado no fluxograma na Figura 2. 


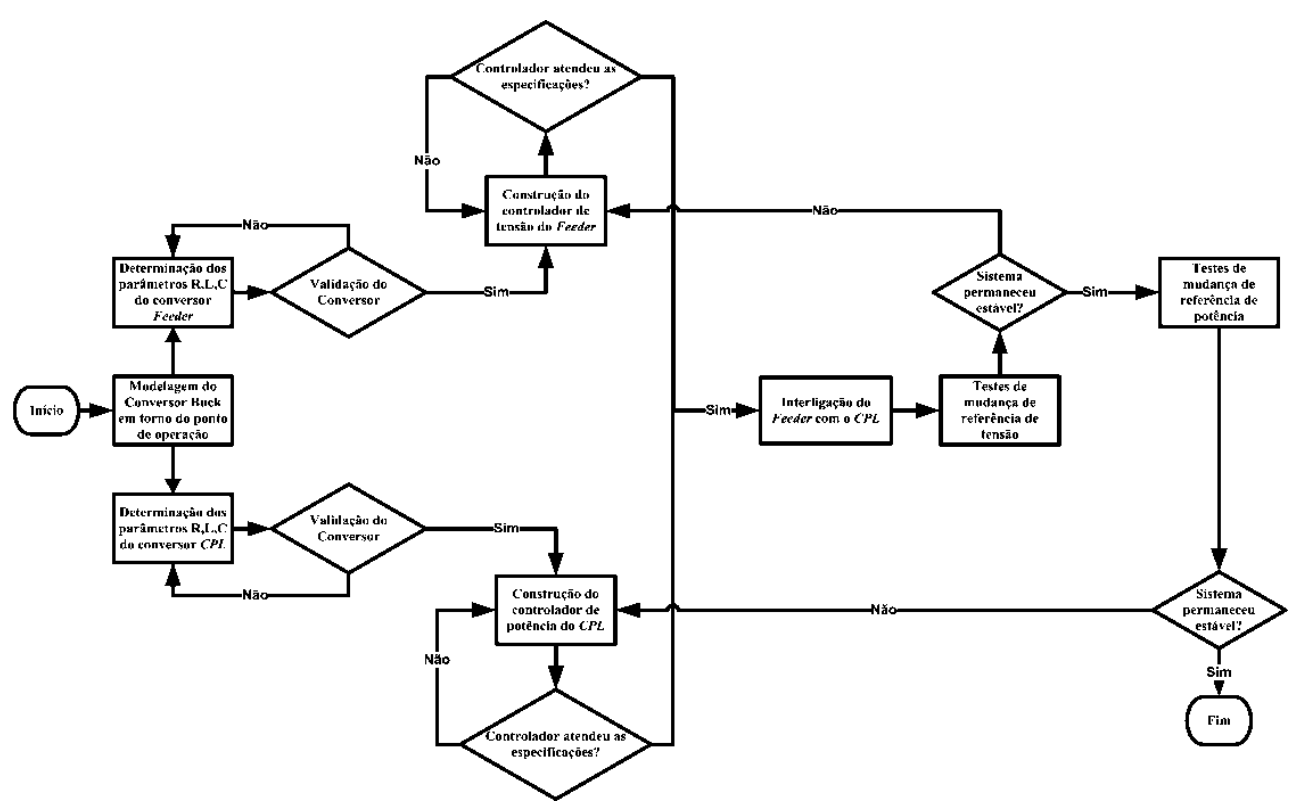

Figura 2: Fluxograma do projeto

\subsection{Especificações do conversor Buck}

Para o projeto do conversor Feeder mostrado na Figura 1a, utiliza-se as equações de relações estáticas para a determinação de parâmetros do conversor, ver em [10]. Para o projeto do Feeder e do CPL utilizou-se um indutor de $1 \mathrm{mH}$. O Feeder opera a uma frequência de chaveamento de $1 \mathrm{KHz}$, enquanto o $C P L$ opera com $2 \mathrm{KHz}$. A relação entre tensão $V_{o}$ na saída e $V_{i}$ que caracteriza um Feeder é apresentada na Eq.(12).

$$
V_{o}=D V_{i}
$$

Para o $C P L$ utilizou-se uma potência de base com $D=1$, desse modo obteve-se a Eq.(13).

$$
P_{b}=\frac{V_{i}^{2}}{R}
$$

Então, a potência em $p u$ na saída do circuito é dada pela Eq.(14)

$$
P_{o}=D^{2}
$$

Dessa forma, as seguintes relações foram utilizadas para a determinação dos parâmetros, ver em [10]:

$$
\begin{gathered}
\Delta I_{o}=\frac{V_{i} D(1-D)}{f L} \\
R \leq \frac{f L}{1-D} \\
C \geq \frac{1-D}{8 f^{2} L\left(\Delta V_{o} / V_{o}\right)}
\end{gathered}
$$

Utilizando as Eq.(12)-(17) determinou-se os parâmetros dos conversores, como mostrado na Tabela 1. 
Tabela 1: Parâmetros dos conversores Buck

\begin{tabular}{l|c|c|c|l|c|c|c}
\hline \multicolumn{1}{c}{ Feeder } & \multicolumn{5}{c}{ CPL } \\
\hline \multicolumn{1}{c}{ Parâmetros } & Símbolo & Valor & Unidade & Parâmetros & Símbolo & Valor & Unidade \\
\hline Tensão de entrada & $V_{i 1}$ & 15,0 & $V$ & Tensão de entrada & $V_{i 2}$ & 12,0 & $V$ \\
Duty Cycle & $D_{1}$ & 0,8 & - & Duty Cycle & $D_{2}$ & 0,6 & - \\
Frequência de chaveamento & $f_{1}$ & 1,0 & $K H z$ & Frequência de chaveamento & $f_{2}$ & 2,0 & $K H z$ \\
Resistência de Carga & $R_{1}$ & 2,0 & $\Omega$ & Resistência de Carga & $R_{2}$ & 2,0 & $\Omega$ \\
Indutor & $L_{1}$ & 1,0 & $m H$ & Indutor & $L_{2}$ & 1,0 & $m H$ \\
Capacitor & $C_{1}$ & 2,0 & $m F$ & Capacitor & $C_{2}$ & 2,0 & $m F$ \\
Tensão de saída & $V_{o 1}$ & 12,0 & $V$ & Potência de saída & $P_{o 2}$ & 0,36 & $p u$ \\
\hline
\end{tabular}

\subsection{Projeto dos controladores do Feeder}

O projeto do controlador do Feeder será desenvolvido neste estudo por meio de duas metodologias clássicas: Alocação de Polos $(A P)$ e Lugar Geométrico das Raízes $(L G R)$ [1]. Para a realização deste projeto, será utilizado a estrutura de controle PID com realimentação de tensão, como mostrado na Figura 3.

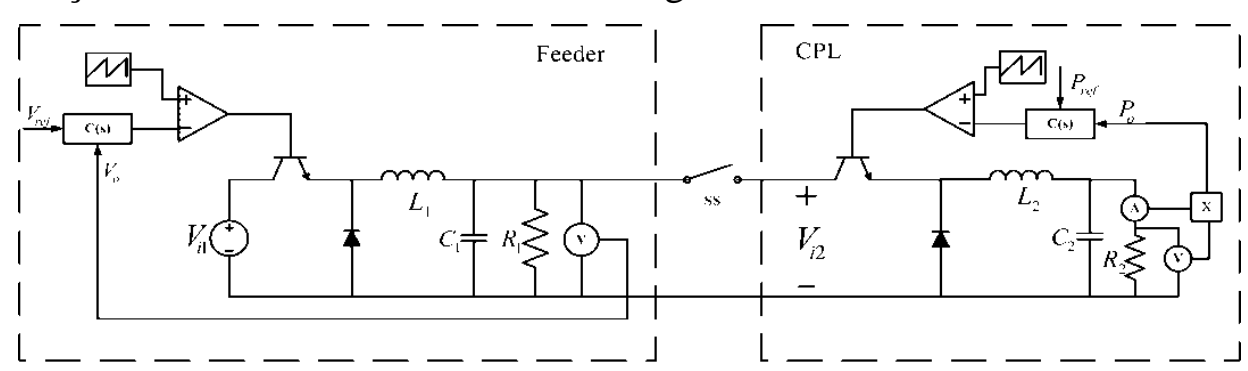

Figura 3: Microrrede interligada pela chave estática (SS, Static Switch)

O projeto do controlador do Feeder foi desenvolvido para atender as especificações de tempo de acomodação $t_{s s}$, overshoot ovs e erro em regime permanente ao degrau $e_{s s}$, conforme apresenta as seguintes especificações desejadas.

$$
\left\{\begin{array}{l}
t_{s s} \leq 2 m s \\
o v s \leq 5 \% \\
e_{s s}=0
\end{array}\right.
$$

Para as especificações dadas na Eq.(18), foram calculados os parâmetros de amortecimento $\zeta$ e frequência natural $\omega_{n}$, conforme apresenta a Eq.(19).

$$
\left\{\begin{array}{l}
\text { ovs }=100 e^{-\frac{\zeta \pi}{\sqrt{1-\zeta^{2}}}} \\
t_{s s}=\frac{4,3}{\zeta \omega_{n}}\left(e_{s s}=1 \%\right)
\end{array}\right.
$$

Utilizando a metodologia de $A P$, obtêm-se um polinômio desejado para o sistema Feeder em malha fechada, considerando as especificações de desempenho desejadas, conforme apresenta a Eq.(20).

$$
\Delta_{d}(s)=\left(s+p_{o}\right)\left(s^{2}+2 \zeta \omega_{n} s+\omega_{n}^{2}\right)
$$

Onde $p_{o}$ é o polo auxiliar do sistema. Com o polinômio característico em malha fechada dado na Eq.(21)

$$
\Delta_{c}(s)=1+C(s) G(s)
$$


Onde, $G(s)$ é a função de transferência do sistema dado pela Eq.(6), com saída em tensão mostrada por meio da Eq.(8) utilizando a estrutura PID com função de transferência dada por meio da função de transferência mostrada na Eq.(22).

$$
\mathrm{C}(\mathrm{s})=\frac{K_{d} s^{2}+K_{p} s+K_{i}}{s}
$$

Desse modo, os ganhos do controlador utilizando a metodologia $A P$, são dados por meio da Eq.(23).

$$
\left\{\begin{array}{l}
K_{p}=\frac{L C\left(\omega_{n}^{2}+2 \zeta \omega_{n}\right)-1}{V_{i}} \\
K_{i}=\frac{L C p_{o} \omega_{n}^{2}}{V_{i}} \\
K_{d}=\frac{L C}{V_{i}}\left(2 \zeta \omega_{n}+p_{o}-\frac{1}{R C}\right)
\end{array}\right.
$$

Já, utilizando a metodologia do $L G R$, foi estabelecido a região onde o controlador irá atuar por meio do uso das especificações solicitadas. Através da comparação das duas metodologias, foi calculado os ganhos do controlador e mostrado na Tabela 2.

Tabela 2: Ganhos dos controladores do Feeder

\begin{tabular}{c|c|c}
\hline Parâmetro & Alocação de Polo & Lugar Geométrico das Raízes \\
\hline$K_{p}$ & $-0,0042$ & 0,0244 \\
$K_{i}$ & 13,9265 & 15,7153 \\
$K_{d}$ & $1,2 \cdot 10^{-4}$ & $6,3 \cdot 10^{-5}$ \\
\hline
\end{tabular}

\subsection{Projeto do controlador do $C P L$}

O projeto do controlador do $C P L$ foi realizado utilizando a metodologia $A P$. Utilizou-se a realimentação de potência realizando essa medida de maneira indireta, como mostrado na Figura 3. Os procedimentos utilizados são análogos aos mostrados na seção anterior para o projeto do Feeder. Os ganhos calculados para o controlador do $C P L$ são apresentados na Tabela 3.

\begin{tabular}{c|c} 
Tabela 3: Ganhos do controlador do & $C P L$ \\
\hline Parâmetro & Alocação de Polo \\
\hline$K_{p}$ & 0,2759 \\
$K_{i}$ & 258,2576 \\
$K_{d}$ & 0,0023 \\
\hline
\end{tabular}

\section{$4 \quad$ Resultados}

\subsection{Variação de referência de tensão do Feeder conectado ao CPL}

Neste teste será verificado o comportamento do Feeder conectado ao CPL para uma variação de referência de tensão do Feeder. Foi analisado o desempenho dos controladores. Observa-se na Figura 4b que o $C P L$ torna-se mais oscilatório com a variação de tensão do Feeder, mostrado na Figura 4a, em decorrência da alteração de tensão de operação, onde o $C P L$ não foi projetado. 


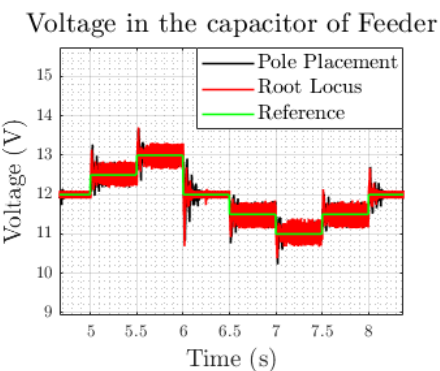

(a)

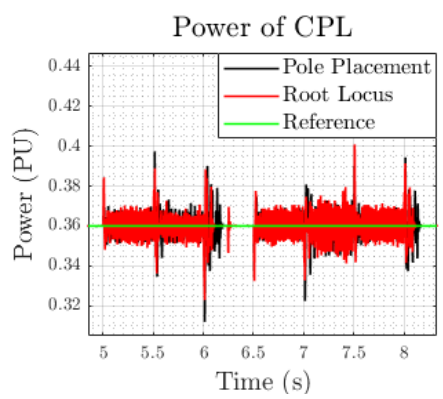

(b)

Figura 4: (a) Para variação de tensão no Feeder (b) a potência na saída do $C P L$

O controlador Feeder construído pela metodologia de $L G R$ apresentou melhor desempenho na estabilidade do sistema em relação ao $A P$, apresentanto menor oscilação na variação de tensão de referência do Feeder. No gráfico de índice de desemepnho mostrado na Figura 5a observa-se que o desepenho do controlador $L G R$ foi superior ao do $A P$ no ponto de operação e embora, perca desempenho fora do ponto de operação, continua com bom desempenho em relação ao controlador projetado por $A P$.

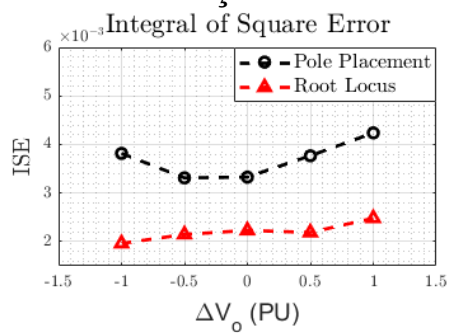

(a)

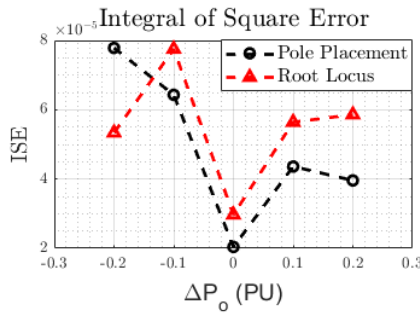

(b)

Figura 5: Índice de desemepnho do controlador do Feeder para (a) variação de referência de tensão e (b) variação de referência de potência.

\subsection{Variação de referência de potência do $C P L$ alimentado pelo Feeder}

Neste teste será verificado o comportamento do $C P L$ para uma variação de referência no valor da potência do mesmo. Deste modo, foi possível analisar o desempenho do controlador de tensão do conversor Feeder projetado. Observa-se na Figura 6a que o controlador do Feeder consegue regular a tensão dentro das especificações de desempenho desejadas. Quando ocorre uma vairação de referência de potência no $C P L$, observa-se os efeitos das oscilações indensejadas no barramento $\mathrm{CC}$, contudo o controlador do Feeder, pelas duas metodologias regulam o sistema mantendo a microrrede estável.

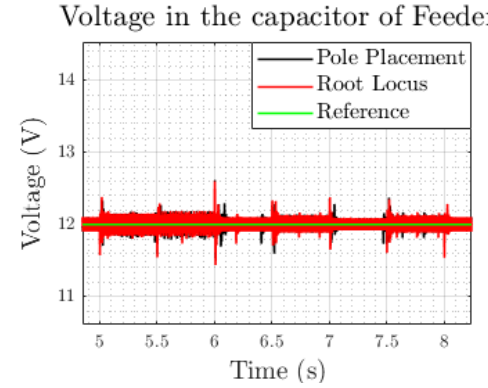

(a)

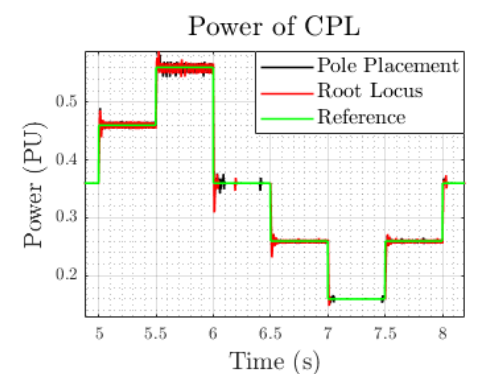

(b)

Figura 6: (a) Comportamento do Feeder para (b) variação de referência de potência

O controlador do Feeder construído pela metodologia de $A P$ apresentou melhor desempenho que o controlador projetado pela metodologia $L G R$, apresentando menor oscilação na variação de potência de referência do $C P L$. Na Figura 5 b é apresentado o índice de desemepenho, para variações de potência de referência do $C P L$. Observa-se na 
Figura $5 \mathrm{~b}$ que o desepenho do controlador projetado pela metodologia de $A P$ foi superior ao desempenho do controlador projetado pela metodologia de $L G R$ no ponto de operação, contudo, retirando o $C P L$ do ponto de operação do sistema, o Feeder projetado por $A P$ perde desempenho assim como o projetado por $L G R$.

\section{Conclusão}

O trabalho propôs apresentar a modelagem do conversor Buck funcionando como um Feeder e como um CPL. Dessa forma, através de estratégias de controle clásicas, projetouse controladores de tensão e potência para o sistema. Após devidamente projetados os controladores do Feeder e do $C P L$, foi feita a conexão entre os mesmos, afim de investigar oscilações decorrentes de variações de potência no $C P L$, o que impacta diretamente na estabilidade do sistema conectado para a variação de referência de potêcia e tensão. Dessa forma, observou-se que as metodologias de controle clásicas, são eficazes para o funcionamento adequado do sistema, sendo que o controlador por $L G R$ apresentou melhor desempenho para uma variação de referência de tensão enquanto que o controlador projetado por $A P$ apresentou melhor desempenho na mitigação de oscilações devido a variação de referência de potência no $C P L$.

\section{Referências}

1. S. Singh; A.R. Gautam; D. Fulwani. Constant Power Load and their effects in DC Distribued Power Systems: A Review. Renewable and Sustaintable Energy Reviws. 2017,Vol.72, pág. 407-421.

2. A.T. Elsayed; A.A. Mohamed; O.A. Mohammed. DC microgrids and distribution systems: A overview. Electric Power Systems Research. 2015, Vol. 2019, pág. 407-417.

3. L.A. Maccari Jr; V.F. Montagner; A.A. Ferreira. A Linear Quadratic Control Applied to Buck Converters with H-Infinity Constraints. Brazilian Power Electronics Conference.2013, pág. 339344.

4. S.Y. Chae; B.C. Hyun; W.S. Kim; B.H. Cho. Digital Load Current Feed-Forward Control Method for a DC-DC Converter. Twenty-Third Annual IEEE Applied Power Electronics Conference and Exposition Research \& Technology. 2008, pág 498-502.

5. M. Salimi; J. Soltani; G.A. Markadeh; N.R. Abjadi. Adaptive Nonlinear Control of the DC-DC Buck Converters operating in CCM and DCM. International Transaction on Electral Energy Systems.2012, pág 1536-1547.

6. A.Jusoh; H. Baamodi; S. Mekhilef. Active Damping Network in DC Distribued Power System Driven by Photovoltaic System. Solar Energy. 2012, Vol.87, pág. 254-267.

7. L.A. Maccari Jr; R.L. Valle; A.A. Ferreira; P.G. Barbosa; V.F. Montagner. A LQR Design with Rejection of Disturbances and Robustness to Load Variation Applied to a Buck Converter. IEEE Transactions on Power Electronics. 2015, Vol.21, pág. 7-15.

8. R.W. Erickson; D. Maksimmovíc, Fundamentals of Power Eletronics, Kluwer Academic Publishers, New York, 2004.

9. H. Guldemir. Study of Sliding Mode Control of DC-DC Buck Converter. Vol.3, Scientific Research.2011, pág. 401-406.

10. D.W. Hart, Power Electronics, McGraw-Hill, New York, 2011.

11. G.F. Franklin; J.D. Powell; A. Emani-Naeini, Feedback Control of Dunamic Systems, Pearson, New Jersey, 2011. 